MATEC Web of Conferences 3, 01030 (2013)

DOI: $10.1051 /$ matecconf/20130301030

(C) Owned by the authors, published by EDP Sciences, 2013

\title{
Thermal treatment of moroccan phosphogypsum
}

\author{
S. El Issiouy, A. Atbir, S. Mançour-Billah, R. Bellajrou, L. Boukbir, and M. El Hadek
}

LGP, Chemical department, Faculty of Sciences, Ibn Zohr University, B.P 8106, Agadir 80000, Morocco

\begin{abstract}
Phosphogypsum (PG) is produced as a by-product during treatment of phosphate rock with sulphuric acid to produce phosphoric acid according to the following simplified reaction:

$\mathrm{Ca}_{10}\left(\mathrm{PO}_{2}\right)_{6} \mathrm{~F}_{2}+10 \mathrm{H}_{2} \mathrm{SO}_{4}+20 \mathrm{H}_{2} \mathrm{O} \rightarrow 6 \mathrm{à}_{3} \mathrm{PO}_{4} \mathrm{C}+2 \mathrm{HF}+10\left(\mathrm{CaSO}_{4} \cdot 2 \mathrm{H}_{2} \mathrm{O}\right)$

Minerai Phosphogypse

PG is mainly $\mathrm{CaSO}_{4} \cdot 2 \mathrm{H}_{2} \mathrm{O}$ but also contains impurities such as free phosphoric acid, phosphates, fluorides and organic matter that adhere to the surface of the gypsum crystals. Phosphogypsum is discharged directly to the Sea or into the natural evaporation ponds. Previous studies have focused on reducing impurity levels in PG. Phosphogypsum impurities can be removed by simple techniques. Washing with water removes the soluble impurities. By cons, other contaminants (radioactive elements, heavy metals ...) a specific treatment method required a complex technique where the treatment is likely to be expensive. In this study, we studied purification of phosphogypsum using water and the thermal behavior of the natural gypsum and Moroccan phosphogypsum to calculation of parameters for drying and dehydration reactions. Also, the effects of different heating temperature on the course of dehydration are investigated.
\end{abstract}

\section{Introduction}

Le phosphogypse est un sous produit de l'industrie phosphatière. Il est généré au stade de la fabrication de l'acide phosphorique. Le procédé consiste à attaquer le minerai de phosphate naturel par l'acide sulfurique. Ce procédé permet d'obtenir un phosphogypse sous forme de dihydrate $\left(\mathrm{CaSO}_{4} \cdot 2 \mathrm{H}_{2} \mathrm{O}\right)$ alors que d'autres procédés conduisent à l'obtention d'un semihydrate $\left(\mathrm{CaSO}_{4} \cdot 0,5 \mathrm{H}_{2} \mathrm{O}\right)$ ou d'une anhydrite $\left(\mathrm{CaSO}_{4}\right)$. Le procédé au dihydrate est celui adopté au Maroc. La fabrication d'une tonne d'acide phosphorique engendre 5 tonnes de phosphogypse. Ainsi, les unités de fabrication de l'acide phosphorique, de par le monde, produisent annuellement environ 150 millions de tonnes de phosphogypse. Une partie seulement de cette quantité trouve une utilisation industrielle ou commerciale, le reste, estimé à 130 millions de tonnes, constitue un apport à l'environnement, qui se traduit, essentiellement, par un stockage au sol ou une décharge dans les eaux de mer ou de surface sans aucun traitement au préalable.

L'intérêt croissant porté aux problèmes d'environnement a amené la prise en considération de nuisances, réelles ou fictives, induites par ces pratiques. En effet, le phosphogypse contient généralement des composants nuisibles provenant soit de l'origine du phosphate, soit du procédé de fabrication. Pour le Maroc notamment, cette problématique est de première importance $\mathrm{du}$ fait qu'il est le premier exportateur mondial du phosphate et le troisième producteur avec une production de 30 millions de tonnes par an.

L'importance du sulfate de calcium dihydraté $\left(\mathrm{CaSO}_{4} \cdot 2 \mathrm{H}_{2} \mathrm{O}\right)$ dans les domaines industriel et agricole a suscité l'intérêt des chercheurs pour prouver que le phosphogypse n'est plus un déchet, mais un co-produit de la fabrication de l'acide phosphorique susceptible d'être valorisé afin de remplacer le gypse naturel dans la plupart de ses applications telles que celles qui concernent les industries plâtrière, cimentière, sucrière, papetière et agricoles [1-6].

\subsection{Traitement physicochimique du phosphogypse}

Dans le phosphogypse, on trouve du quartz et/ou des silicates, des fluorures, des phosphates, des matières organiques et des minéraux de fer et d'aluminium.

- Impuretés solubles : ce sont des sels ou acides non éliminés par le lavage du procédé $\left(\mathrm{P}_{2} \mathrm{O}_{5}\right.$ et fluor principalement solubles). Ces acides confèrent au phosphogypse un $\mathrm{pH}$ assez faible (généralement entre 2 et 4).

- Impuretés insolubles : ces impuretés insolubles proviennent :

$\checkmark$ Du minéral sans transformation pendant l'attaque phosphorique (silice, minerai non attaqué, carbone organique ....).

$\checkmark$ Des réactions secondaires dans le milieu d'attaque: $\mathrm{P}_{2} \mathrm{O}_{5}$ syncristallisé et éléments traces, principalement des métaux lourds. 
Les minerais de phosphate naturel utilisés pour la fabrication de l'acide phosphorique contiennent également des traces d'éléments radioactifs d'origine naturelle. Ces radioéléments peuvent se retrouver pour partie dans le phosphogypse.

Singh a montré que le phosphogypse peut être purifié par tamisage. Les grosses particules, représentant une fraction de 10 à $15 \%$, sont riches en impuretés $\mathrm{P}_{2} \mathrm{O}_{5}, \mathrm{~F}$, matière organique, $\mathrm{Na}_{2} \mathrm{O}$ et $\mathrm{K}_{2} \mathrm{O}$ [7]. En plus, le traitement du phosphogypse par l'eau et l'hydrocyclone réduit considérablement la teneur de ces impuretés.

Al-Masri a étudié les caractéristiques du mécanisme de transfert et de distribution des éléments $\mathrm{Cu}, \mathrm{Cd}, \mathrm{Zn}$ et $\mathrm{U}$ lors du lavage du phosphogypse Syrien [8]. Le lavage du phosphogypse avec l'eau et l'acide sulfurique dilué permet facilement l'élimination d'une partie du $\mathrm{Cu}$ et du Zn [8]. Par contre, uniquement $21 \%$ d'uranium, présent dans le phosphogypse, est soluble dans l'eau.

Tafu et Chohji ont montré que les ions fluorure du phosphogypse peuvent être fixés par l'hydrogénophosphate de calcium dihydraté (DCPD) $\mathrm{CaHPO}_{4} \cdot 2 \mathrm{H}_{2} \mathrm{O}$ en formant des composés stables de fluorure tels que les fluorapatites selon la réaction [9]: $10 \mathrm{CaHPO}_{4} \cdot 2 \mathrm{H}_{2} \mathrm{O}+2 \mathrm{~F}^{-} \rightarrow \mathrm{Ca}_{10}\left(\mathrm{PO}_{4}\right)_{6} \mathrm{~F}_{2}+6 \mathrm{H}^{+}+4 \mathrm{HPO}_{4}{ }^{2-}$ $+20 \mathrm{H}_{2} \mathrm{O}$

Chohji a développé une nouvelle méthode, basée sur les équilibres chimiques, pour dissoudre le phosphogypse en vue de le purifier et de le réutiliser comme engrais liquides exempt d'ions fluorure [10].

\subsection{Traitement thermique du phosphogypse}

Taher a étudié le traitement thermique du phosphogypse Egyptien à différentes températures afin de l'épurer et d'améliorer sa qualité pour l'adapter à la fabrication du laitier du ciment Portland [11]. Le phosphogypse thermiquement traité s'est avéré qu'il renferme moins d'impuretés en $\mathrm{P}_{2} \mathrm{O}_{5}$, fluorures et matière organique que le phosphogypse non traité.

Généralement toutes les études de déshydratation de $\mathrm{CaSO}_{4} \cdot 2 \mathrm{H}_{2} \mathrm{O}$ employant l'ATD montrent la présence de deux pics endothermiques. Cependant, les valeurs bibliographiques des températures correspondantes ont un écart remarquable : des pics ont été obtenus à 130-180, à $180-215$ ou à $149-169^{\circ} \mathrm{C}$, Sebbahi et Elbeyli [12, 13]. Kacimi a étudié la valorisation du phosphogypse dans la production du ciment et son influence sur la température du traitement du clinker [14]. Il a montré que l'addition du phosphogypse $(10 \%)$ permet une réduction de la température de clinkerisation de $1470^{\circ} \mathrm{C}$ à $1200^{\circ} \mathrm{C}$. Ce qui augmente l'efficacité énergétique de l'usine de ciment de $25 \%$ et par conséquent, prolonge la durée de vie des briques réfractaires du four.

En continuation, nous aborderons dans cette étude une contribution à la purification du phosphogypse marocain et à son traitement thermique afin de contribuer à la valorisation du sous-produit industriel phosphogypse et à protéger l'environnement par son exploitation dans la production du ciment de Portland et par la réduction de l'émission des gaz $\mathrm{CO}_{2}, \mathrm{NO}_{\mathrm{x}}$ et $\mathrm{SO}_{\mathrm{x}}$ dû à la diminution de la température de clinkerisation.

\section{Techniques expérimentales}

\subsection{Produits chimiques utilisés}

Le sulfate de calcium $\left(\mathrm{CaSO}_{4} \cdot 2 \mathrm{H}_{2} \mathrm{O}\right)$ utilisé dans ce travail est un produit Merck pour analyse de pureté égale à $99 \%$. Le phosphogypse utilisé dans ce travail provient des unités industrielles de production de l'acide phosphorique de JorfLasfar situé à $30 \mathrm{Km}$ au sud d'Eljadida. Sa composition chimique est présentée dans le tableau 1.

Tableau 1. Composition chimique du phosphogypse [12].

\begin{tabular}{|c|c|c|c|c|c|c|c|c|c|c|}
\hline Elém. & $\mathrm{H}_{2} \mathrm{O}$ & $\mathrm{SO}_{3}$ & $\mathrm{CaO}$ & $\mathrm{SiO}_{2}$ & $\mathrm{CO}_{2}$ & $\mathrm{Fe}_{2} \mathrm{O}_{3}$ & $\mathrm{Al}_{2} \mathrm{O}_{3}$ & $\mathrm{P}_{2} \mathrm{O}_{5}$ & $\mathrm{MgO}$ & $\mathrm{F}$ \\
\hline $\begin{array}{c}\% \\
\text { Mass. }\end{array}$ & 3.5 & 42.9 & 30.5 & 9.5 & 4.5 & 0.9 & 2.8 & 0.5 & 0.3 & 0.15 \\
\hline
\end{tabular}

Le réacteur utilisé est une jaquette thermostatique en verre Pyrex et de volume $500 \mathrm{ml}$. La température du réacteur est fixée à l'aide d'un thermostat à circulation d'eau, type Lauda RK20 susceptible de fixer des températures entre -40 et $150^{\circ} \mathrm{C}$ avec une précision de $0,1^{\circ} \mathrm{C}$.

\subsection{Mesure de conductivité électrique}

Nous avons utilisé un conductimètre WTW modèle LF 530 caractérisé par une gamme de mesure très large de 0 à $1999 \mathrm{mS} \mathrm{cm}^{-1}$. Les cellules conductimétriques utilisées sont de type TACUSSEL de constantes 0,$1 ; 1$ et $10 \mathrm{~cm}^{-1}$.

\subsection{Analyse thermique différentielle couplée à l'analyse thermogravimétrie (ATD-ATG)}

L'appareil utilisé est de type SHIMADZU ATD-ATG couplé, série DTG-60/60H. Il est constitué d'une balance à haute précision avec une nacelle en aluminium (pour éviter les réactions parasites) et d'un four pouvant atteindre $1000^{\circ} \mathrm{C}$. L'ensemble, piloté par un ordinateur, permet le tracé simultané de la courbe de variation des masses de l'échantillon et de la courbe d'analyse thermique différentielle. L'appareil permet de travailler sous atmosphère contrôlée.

\section{Résultats expérimentaux}

\subsection{Lavage du phosphogypse}

Le procédé du lavage avec l'eau est réalisé de la façon suivante : à une certaine masse de $10 \mathrm{~g}$ de phosphogypse introduite dans un bêcher, on ajoute $20 \mathrm{ml}$ d'eau. L'ensemble est agité magnétiquement pendant $30 \mathrm{~min}$ à une température constante, $25^{\circ} \mathrm{C}$.

Ensuite, la solution est filtrée sous vide. Le filtrat est recueilli afin d'effectuer les mesures de $\mathrm{pH}$ et de la conductivité électrique. La matière solide retenue par le verre fritté (porosité 3) a été récupérée. La procédure de lavage est renouvelée plusieurs fois jusqu'à ce que les valeurs constantes de $\mathrm{pH}$ et de conductivité électrique du gypse naturel pur, pris comme référence, soient atteintes. 
La figure 1 donne un exemple de courbe obtenue lorsque le nombre de lavages est égal à quatorze.

Afin de faire une comparaison, nous avons mesuré et représenté sur la même figure 1 le $\mathrm{pH}$ et la conductivité d'une solution saturée uniquement de $\mathrm{CaSO}_{4} \cdot 2 \mathrm{H}_{2} \mathrm{O}$ pur. La dissolution de phosphogypse entraine une diminution rapide de la conductivité électrique dès le premier instant de la mise en solution. Juste après cinq lavages, la conductivité chute de 5,25 pour se stabiliser à $2,1 \mathrm{mS} / \mathrm{cm}$ (valeur correspondant à $\mathrm{CaSO}_{4} \cdot 2 \mathrm{H}_{2} \mathrm{O}$ pur).

Par contre, le $\mathrm{pH}$ de la solution du phosphogypse augmente de 2,7 à 5,95 (valeur correspondant à CaSO4.2H2O pur). Dans ce cas, ce n'est qu'après le dixième lavage que l'évolution du $\mathrm{pH}$ devient négligeable.

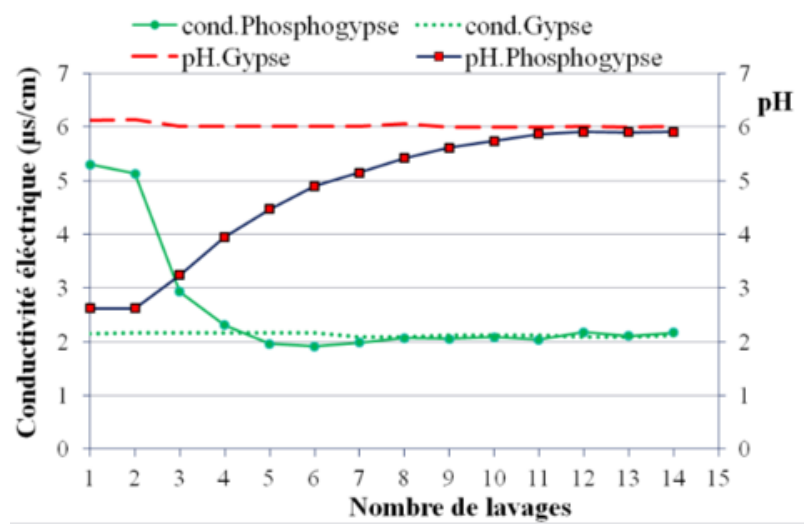

Figure 1. Evolution de la conductivité électrique et du $\mathrm{pH}$ pendant le lavage du phosphogypse.

\subsection{Déshydratation isotherme du phosphogypse}

Parmi les techniques de purification, il a été constaté que le lavage avec l'eau, permet d'enlever les impuretés solubles du phosphogypse. Toutefois, le traitement thermique conduit à optimiser le taux d'hydratation, l'élimination des gaz indésirables et les impuretés volatiles.

Les paramètres temps et température sont indispensables pour contrôler le comportement thermique des matériaux. Dans cette partie, nous proposons d'étudier l'évolution du temps nécessaire pour la déshydratation isotherme du gypse naturel, du phosphogypse et du phosphogypse lavé à 80, 100, 120, 140 et à $160{ }^{\circ} \mathrm{C}$.

Pour réaliser cette expérience, on dispose de trois échantillons; le gypse naturel, le phosphogypse et le phosphogypse lavé de masses précises. L'ensemble est placé dans l'étuve à l'une des températures précitées pendant une durée de $20 \mathrm{~min}$. On fait sortir les échantillons, après un refroidissement dans un dessiccateur pendant une durée d'environs $10 \mathrm{~min}$, on effectue la pesée dans une balance électronique de précision $\Delta m=10^{-4} \mathrm{~g}$. La procédure est répétée jusqu'à ce que le poids de tous les échantillons se stabilise. La figure 2 schématise le diagramme de procédé de séchage isotherme.

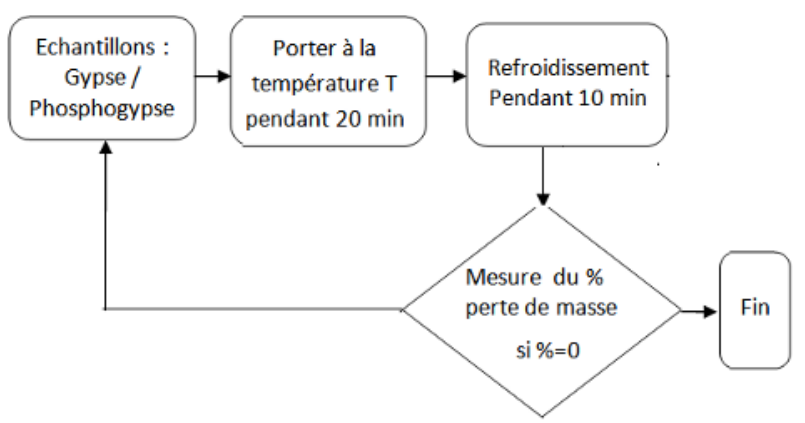

Figure 2. Diagramme du procédé de séchage isotherme.

Le pourcentage de perte au cours de la déshydratation est calculé par l'intermédiaire des données de poids de l'échantillon avant et après séchage. Les résultats obtenus sont représentés dans les figures 3, 4 et 5 .

L'échantillon du gypse naturel et non lavé était considéré comme référence. Le tableau 1 rassemble le temps nécessaire au traitement thermique et le pourcentage de perte en masse pour les différents échantillons.

Les figures 3,4 et 5 donnent, à température constante $80,100,120140$ ou $160^{\circ} \mathrm{C}$, l'évolution en fonction du temps de la perte en masse du gypse naturel, du phosphogypse et du phosphogypse lavé. Aucune perte en masse n'a été observée pendant le chauffage à $80{ }^{\circ} \mathrm{C}$ des échantillons du gypse naturel (fig. 3) et du phosphogypse lavé (fig. 5). Par contre, une perte de masse (environ $10 \%$ ) a été observée pour le traitement thermique du phosphogypse à $80^{\circ} \mathrm{C}$ (fig. 4).

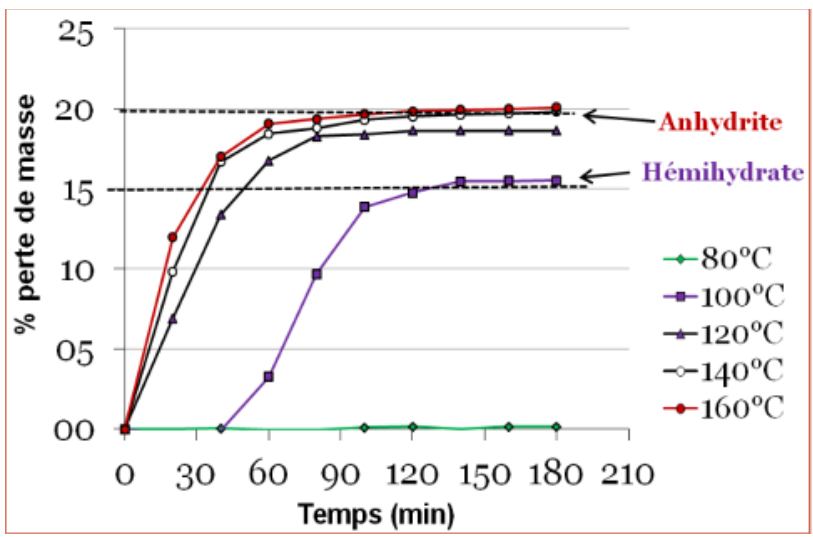

Figure 3. Cinétique de séchage isotherme de gypse naturel.

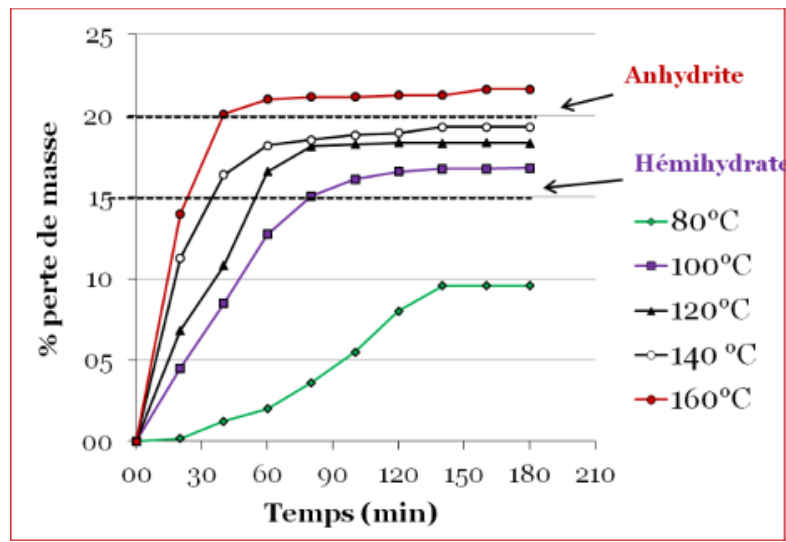

Figure 4. Cinétique de séchage isotherme du phosphogypse. 


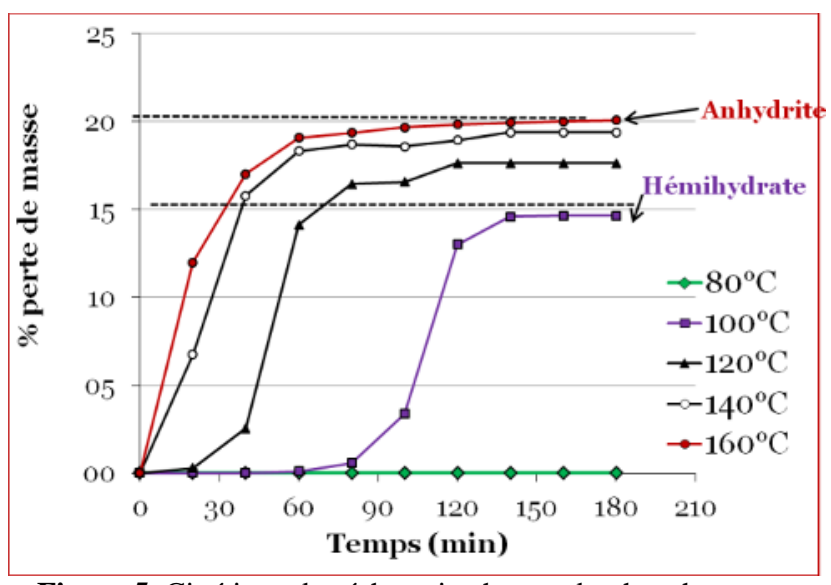

Figure 5. Cinétique de séchage isotherme du phosphogypse lavé.

D'autre part, il apparaît que la déshydratation à $100{ }^{\circ} \mathrm{C} \mathrm{du}$ gypse naturel et du phosphogypse lavé atteint une valeur d'environ $15 \%$ de perte en masse après deux heures de traitement thermique. Cette perte correspond à la transformation du gypse dihydraté au hémihydrate :

$\mathrm{CaSO}_{4} \cdot 2 \mathrm{H}_{2} \mathrm{O} \rightarrow \mathrm{CaSO}_{4} \cdot 0,5 \mathrm{H}_{2} \mathrm{O}+1,5 \mathrm{H}_{2} \mathrm{O}$

La déshydratation maximale (20\% de perte de masse) n'est obtenue qu'après chauffage des échantillons plus d'une heure et d'une heure $30 \mathrm{~min}$ respectivement à 140 ${ }^{\circ} \mathrm{C}$ et à $120^{\circ} \mathrm{C}$.

La cinétique de déshydratation des différents échantillons est résumée dans le tableau 1. En générale, cette cinétique est plus rapide pour le phosphogypse à $\mathrm{T}=80,100$ et $120^{\circ} \mathrm{C}$. Par contre, on obtient la même cinétique pour les hautes températures $\mathrm{T}=140$ et $160^{\circ} \mathrm{C}$.

Tableau 2. Traitement thermique isotherme du gypse naturel, du phosphogypse et du phosphogypse lavé.

\begin{tabular}{|c|c|c|c|c|}
\hline $\begin{array}{c}\text { Température } \\
\left({ }^{\circ} \mathrm{C}\right)\end{array}$ & & $\begin{array}{l}\text { Gypse } \\
\text { naturel }\end{array}$ & $\begin{array}{l}\text { Phospho- } \\
\text { gypse }\end{array}$ & $\begin{array}{l}\text { Phospho- } \\
\text { gypse lavé }\end{array}$ \\
\hline \multirow[t]{2}{*}{80} & $\begin{array}{l}\% \text { perte } \\
\text { masse }\end{array}$ & 0 & 10 & 0 \\
\hline & Temps(min) & 180 & 140 & 180 \\
\hline \multirow[t]{2}{*}{100} & $\begin{array}{l}\% \text { perte } \\
\text { masse }\end{array}$ & 15,1 & 17 & 14,98 \\
\hline & Temps(min) & 140 & 145 & 141 \\
\hline \multirow[t]{2}{*}{120} & $\begin{array}{l}\% \text { perte } \\
\text { masse }\end{array}$ & 18,1 & 18,2 & 18 \\
\hline & Temps(min) & 138 & 140 & 140 \\
\hline \multirow[t]{2}{*}{140} & $\begin{array}{l}\% \text { perte } \\
\text { masse }\end{array}$ & 19,5 & 19,95 & 19,65 \\
\hline & Temps(min) & 135 & 138 & 135 \\
\hline \multirow[t]{2}{*}{160} & $\begin{array}{l}\% \text { perte } \\
\text { masse }\end{array}$ & 20 & 22,5 & 20,1 \\
\hline & Temps(min) & 130 & 120 & 120 \\
\hline
\end{tabular}

\subsection{Analyses thermique différentielle thermogravimétriques ATD/ATG}

Les échantillons à analyser sont séchés auparavant pendant 24 heures à $40^{\circ} \mathrm{C}$, l'analyse s'effectue sur environ $20 \mathrm{mg}$ de produit. La montée en température étudiée varie de l'ambiant jusqu'à $600^{\circ} \mathrm{C}$ avec une vitesse de 2 , de 5 ou de $10^{\circ} \mathrm{C} / \mathrm{min}$ pour les échantillons contenant le gypse, phosphogypse non lavé et le phosphogypse lavé.

La déshydratation du gypse pur (produit Merck) se déroule en plusieurs étapes, fig.6. On retrouve (comme attendu) les pics relatifs à la déshydratation du gypse de façon distincte et précise. Deux pics endothermiques à $125^{\circ} \mathrm{C}$ et à $140^{\circ} \mathrm{C}$ correspondant à la déshydratation du gypse en semihydrate (plâtre) puis en anhydrite III traduisant une modification de la structure cristalline (monoclinique : gypse $\rightarrow$ hexagonale : anhydrite III): * 1 er pic à $125^{\circ} \mathrm{C}: \mathrm{CaSO}_{4} \cdot 2 \mathrm{H}_{2} \mathrm{O} \rightarrow \mathrm{CaSO}_{4} \cdot 0,5 \mathrm{H}_{2} \mathrm{O}+$ $1,5 \mathrm{H}_{2} \mathrm{O}$

* 2ème pic à $140^{\circ} \mathrm{C}: \mathrm{CaSO}_{4} \cdot 0,5 \mathrm{H}_{2} \mathrm{O} \rightarrow \mathrm{CaSO}_{4} \cdot \mathrm{III}+$ $0,5 \mathrm{H}_{2} \mathrm{O}$

* 3ème pic exothermique entre $320-450^{\circ} \mathrm{C}$ correspond à une transformation cristallographique (hexagonale $\rightarrow$ orthorhombique) :

$$
\mathrm{CaSO}_{4} \text {.III } \rightarrow \mathrm{CaSO}_{4} \text {.II }
$$

En ce qui concerne le phosphogypse, lavé ou non, les pics endothermiques relatifs à la déshydratation du gypse en semihydrate puis du semihydrate en anhydrite III ne sont pas dissociés (fig.7).

Nous avons ensuite étudié l'influence de la vitesse de chauffe de 2 , de 5 ou de $10^{\circ} \mathrm{C} / \mathrm{min}$ afin d'essayer de séparer les deux réactions de déshydratation du phosphogypse (fig.8)

Nous remarquons qu'en accroissant la vitesse de chauffe, les surfaces des pics s'élargissent et les températures correspondantes s'étalent légèrement vers les hautes valeurs. Cependant, l'allure des pics du phosphogypse n'a pas été modifiée, un seul pic sans dissociation. Dans tous les cas, la perte en masse se fait en une seule étape. Elle est d'environ 20\% (perte de $2 \mathrm{H}_{2} \mathrm{O}$ ).

Les analyses thermiques conduites par Sebbahi et al. [12] montrent que le phosphogypse se déshydrate d'une manière analogue au gypse : pics endothermiques et exothermiques observés aux mêmes températures, vitesses de déshydratation et pertes de masses identiques. Ces conclusions sont en contradiction avec celles tirées des études effectuées par Guilhot, [15], Bourgier [16] et celles du présent travail.

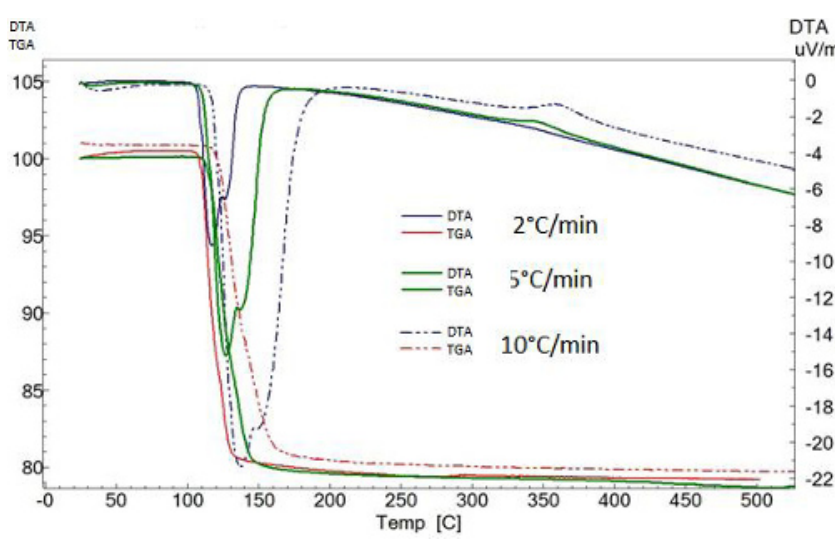

Figure 6. Effet de la vitesse de chauffage $\left(2,5\right.$ et $\left.10^{\circ} \mathrm{C} / \mathrm{min}\right)$ sur le comportement thermique du gypse 


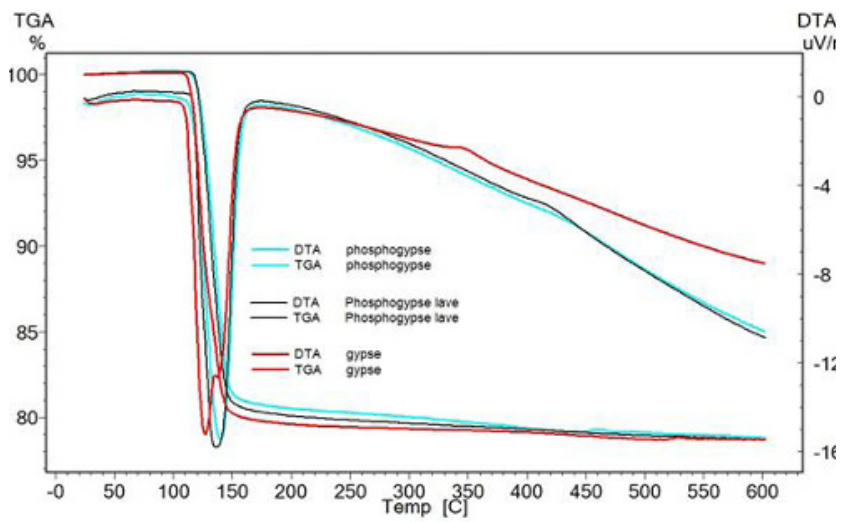

Figure 7. Comparaison du traitement thermique du gypse, phosphogypse et phosphogypse lavé de l'ambiant à $600^{\circ} \mathrm{C}$ sous air $\left(5^{\circ} \mathrm{C} / \mathrm{min}\right)$.

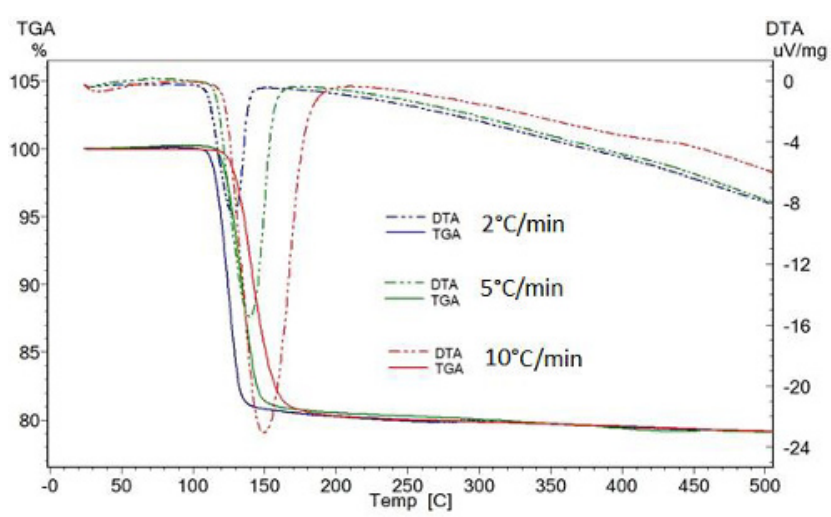

Figure 8. Effet de la vitesse (2, 5 et $10 \mathrm{deg} / \mathrm{min})$ de chauffage sur le comportement thermique de phosphogypse.

\section{Conclusion}

Nous nous sommes intéressés dans un premier temps, à l'étude de la purification du phosphogypse. Les résultats de ce travail montrent que le traitement avec l'eau permet d'enlever les impuretés solubles. Toutefois, le traitement thermique conduit à optimiser l'élimination des gaz indésirables et les impuretés volatiles.

Nous avons établi une courbe d'évolution du $\mathrm{pH}$ et de la conductivité électrique en fonction du nombre de lavages. Cette corrélation peut donc être utilisée comme un guide et outil de référence pour évaluer l'état de qualité et de pureté du phosphogypse Marocain. Selon le mode d'application du phosphogypse, on peut donc prévoir le nombre de lavages appréciable.

On peut finalement conclure, de manière générale, que le comportement thermique du gypse naturel et du phosphogypse lavé est assez semblable. La température et le temps de séchage semblent être des facteurs très influençant sur le traitement thermique du gypse naturel et du phosphogypse lavé. Toutefois, le traitement thermique du phosphogypse non lavé semble différent à cause de la présence des gaz indésirables et d'impuretés volatiles.

\section{Références}

1. E.M. Elkanzi and M.F. Chalabi, Ind. Eng. Chem. Res. 301289 (1991)
2. W.C. Burnett, M.K. Schultz, D.H. Carter J. Environ. Rad. 3233 (1996)

3. A. Aagli, N. Tamer, A. Atbir, L. Boukbir, M.El Hadek, J. Therm. Anal. Cal. 82395 (2005)

4. S.I. Abu-Eishah, A.A. Bani-Kananeh, M.A. Allawzi, Chem. Eng. J. 76197 (2000)

5. J. Mulopo and D. Ikhu-Omoregbe, J. Chem. Eng. Process. Technol. 3 (2012)

6. C. Cárdenas-Escudero et al., J. Hazard. Mater. 196 $431(2011)$

7. J.V. Baird and E.J. Kamprath, Proceedings of the First International Symposium on phosphogypsum, publication FIPR n ${ }^{\circ}$ 01-001-017 133 (1980)

8. A. Davister, Conférence Technique de l'IFA, Marrakech, Maroc (1998)

9. M. Tafu, T. Chohji, J. Eur. Ceram. Soc. 26767 (2006)

10. T. Chohji and M. Tafu, Proceedings of the 6th Tunisian-Japanese Seminar on Culture, Science and Technology, Soussa, Tunisia (2005)

11. M.A. Taher, Resour., Conserv. and Recy. 5228 (2007)

12. S. Sebbahi, M.L.O. Chameikh, F. Sahban, J. Aride, L. Benarafa and L. Belbir, Thermochim. Acta 30269 (1997)

13. I.Y. Elbeyli, E.M. Derun, J. Gülen and S. Piskin, Cem. Concr. Res 331729 (2003)

14. L. Kacimi, A. Simon-Masseron, A. Ghomari, Z. Derriche, J. Haz. Mat. B137 129 (2006) 7

15. B. Guilhot, Gardet, M. Soustelle, J.P. Caspar, Viviers, and Eymery. Rapport interne confidentiel Ecole Nationale Supérieure des Mines de Saint-Etienne Lafarge (1974)

16. V. Bourgier, These, Ecole Nationale Supérieure des Mines de Saint-Etienne (2007) 